\title{
Prevalencia de los dientes supernumerarios en la población infantil asturiana
}

\section{Prevalence of supernumerary teeth in the child population of Asturias}

\author{
Cueto Blanco S*, Pipa Vallejo A**, González García M***, Pipa Muñiz C****, \\ Álvarez Álvarez I*****
}

\section{RESUMEN}

La odontogénesis, que es un proceso extraordinariamente complejo, como el resto del desarrollo embrionario, está expuesta a determinados factores que pueden alterar su normal evolución.

En ocasiones podemos observar un número de dientes adicional a la fórmula dental normal y en ese caso se habla de dientes supernumerarios, hiperodoncia o hipergenesia. Los dientes supernumerarios son más frecuentes en varones que en mujeres. Y no es raro que puedan dar lugar a retención de dientes permanentes o dificultar su erupción en la arcada dentaria. El diagnóstico de hipergenesia se realiza a partir de una exhaustiva exploración clínica con un completo estudio radiográfico, mediante radiografía panorámica, complementado con radiografías periapicales, oclusales, etc. Los dientes supernumerarios se localizan sobretodo en la línea media anterior del maxilar, en cuyo caso hablamos de mesiodens. El mesiodens es el diente supernumerario que se observa con mayor frecuencia. Los dientes supernumerarios son más frecuentes en dentición permanente y más habitual en el maxilar superior que en el maxilar inferior. El abordaje más habitual de los dientes supernumerarios es la exodoncia, aunque en ocasiones se debe complementar con tratamiento ortodóncico.

Palabras clave: Dientes supernumerarios, hipergenesia, hiperodoncia, mesiodens.

\section{SUMMARY}

Odontogenesis, which is an extraordinarily complex process, like rest of embryonic development, is exposed to certain factors that may alter its normal evolution.

Sometimes we see a number of additional teeth to normal dental formula, and if we speak of supernumerary teeth, hyperdontia or hipergenesia. Supernumerary teeth are more common in men than in women.And no wonder that may lead to retention of permanent teeth or hinder its eruption in the dental arch.Hipergenesia diagnosis is made from a though clinical examination with a complete radiographic study by panoramic radiography supplemented with periapical radiographs, occlusal, etc. Supernumerary teethare located mainly in the anterior midline of the maxilla, in which case we speak of mesiodens. The supernumerary tooth mesiodens is seen more often. Supernumerary teeth are more common in permanent dentition and more common in the

* Odontóloga. Máster en Ortodoncia. Escuela de Odontología. Facultad de Medicina. Universidad de Oviedo.

** Profesor Asociado. Escuela de Odontología. Facultad de Medicina. Universidad de Oviedo.

*** Profesor Asociado. Servicio de Cirugía Oral y Maxilofacial del HUCA.

**** Odontóloga. Alumna de Postgrado de Cirugía Oral. Escuela de Odontología. Facultad de Medicina. Universidad de Oviedo.

***** Profesora Asociada. Escuela de Odontología. Facultad de Medicina. Universidad de Oviedo. 
maxilla than the mandibule. The most common approach of supernumerary teeth is extraction, but sometimes must be supplemented by orthodontic treatment.

Key words: Supernumerary teeth, hipergenesia, hyperdontia, mesioden.

Fecha de recepción: 29 de mayo de 2012.

Aceptado para publicación: 11 de junio de 2012.

Cueto Blanco S, Pipa Vallejo A, González García M, Pipa Muñiz C, Álvarez Álvarez I. Prevalencia de los dientes supernumerarios en la población infantil asturiana. Av Odontoestomatol 2013; 29 (3): 131-137.

\section{INTRODUCCIÓN}

La odontogénesis es un proceso complejo que se inicia en estadios tempranos del desarrollo del embrión y se prolonga a lo largo de los primeros años de la vida.

Los dientes se originan a partir de la interacción del epitelio bucal y el mesénquima subyacente derivado de la cresta neural.

Alrededor de la sexta semana de desarrollo, la capa basal del revestimiento epitelial de la cavidad bucal da origen a la lámina dental de donde se originan los esbozos dentales. El epitelio gobierna la diferenciación del estado del esbozo, hasta el momento que esa función es transferida al mesénquima (1).

El desarrollo embriológico dentario está mediado por una serie de señales entre las que podemos destacar factores de crecimiento como: WNT; proteína morfogenética del hueso (BMP); factor de necrosis tumoral (TNF); factor de crecimiento fibroblástico (FGF); el factor secretado Sonic Hedgehog (SHH) y factores de transcripción como MX1 y 2 (2).

Cualquier noxa que actúe sobre la formación de la lámina dental o el desarrollo del germen dentario, puede dar lugar a defectos en el número, forma, tamaño, estructura o color, de los dientes que se están formando en estas etapas tempranas del desarrollo $(3,4)$.

Dentro de las alteraciones de número las hay por defecto o por exceso.
Cuando existen dientes adicionales a la fórmula dental normal o habitual, hablamos de dientes supernumerarios, hiperodoncia o hipergenesia.

En la mayoría de los casos los dientes supernumerarios suelen estar retenidos en los maxilares, aunque también se han descrito este tipo de dientes en la cavidad nasal, en el seno maxilar, en la fisura orbitaria superior o en la orofaringe (5-8).

Los dientes supernumerarios pueden presentarse de forma única, doble o múltiple.

La prevalencia de dientes supernumerarios oscila entre el $0,3 \%, 0,4 \%-0,8 \%$, en dentición primaria (9$12)$, y el $0,76 \%-3,2 \%$ en dentición definitiva (13-16).

También se ha visto en varios estudios una mayor asociación al sexo masculino (17-19). Pueden encontrarse dientes supernumerarios con relativa frecuencia en pacientes con fisura palatina y labio leporino (20).

No es infrecuente la aparición de hiperodoncia en relación con algunos síndromes del tipo de: displasia cleidocraneal, síndrome de Down, síndrome de Gardner, síndrome de Nance-Horan, síndrome orofacial-digital, síndrome trico-rino-falángico, síndrome de Apert, síndrome de Marfán, etc. (21-28).

En función de su morfología, los dientes supernumerarios se clasifican en suplementarios, cuando su tamaño y forma son normales, y en rudimentarios o dismórficos, cuando estos parámetros son anormales, y éstos, a su vez, se subdividen en conoideos, tuberculados o molariformes. 
Estos dientes supernumerarios se localizan fundamentalmente en el maxilar superior frente a la mandíbula. Y dentro del maxilar la ubicación más frecuente es en el sector anterior (29).

Ante signos clínicos como fallos en la erupción dentaria, giroversiones de los dientes vecinos, diastemas de línea media, desplazamientos dentarios, etc., debe sospecharse de la presencia de dientes supernumerarios, pero para la confirmación diagnóstica se requiere un estudio radiográfico, fundamentalmente la radiografía panorámica, además radiografías periapicales, oclusales, etc., aparte de la exploración oral.

Pueden presentarse complicaciones como problemas eruptivos, formación de quistes e incluso lesiones en los dientes no supernumerarios (30).

Por ello, el tratamiento de elección es generalmente la exodoncia, aunque el momento en que debe realizarse es aún controvertido.

Tras la cirugía en la mayoría de los casos se obtiene una razonable solución estética y armonización oclusal de la arcada dentaria; pero en otras situaciones más complejas se precisa un tratamiento ortodóncico con aparatología fija, que en ocasiones incluye la tracción dentaria (31).

El propósito de este estudio es valorar la prevalencia de dientes supernumerarios, el número, la morfología, localización, posiciones más frecuentes, asociación al sexo o a algunos síndromes, la evolución y la necesidad de tratamiento ortodóncico, a causa de esta alteración en la población infantil de Asturias.

\section{MATERIAL Y MÉTODO}

Se revisaron las radiografías panorámicas de todas las historias de pacientes infantiles, cuyas edades comprendían entre los 3 y los 14 años, de la Clínica Universitaria de la Facultad de Odontología de Oviedo entre los años 2006 y 2010, con el objetivo de conocer la prevalencia de dientes supernumerarios en esta población.

Por otra parte, se examinaron las historias de pacientes infantiles intervenidos por supernumerarios en el
Hospital Universitario Central de Asturias (HUCA) durante esas mismas fechas, con el fin de valorar el número, la morfología, localización y posición más frecuentes, así como su asociación al sexo o a algunos síndromes antes comentados.

Se contactó telefónicamente con los pacientes intervenidos por dientes supernumerarios en el HUCA en el periodo establecido y se les realizó una encuesta telefónica con el fin de conocer su evolución y si fue preciso tratamiento ortodóncico (Tabla 1).

\section{RESULTADOS}

De las 324 historias revisadas en la Clínica Universitaria se encontró un diente supernumerario localizado en la línea media de morfología conoidea (mesiodens), por lo que podemos decir que la prevalencia de supernumerarios en población infantil de Asturias es de $0,31 \%$.

De los 28 pacientes intervenidos en el HUCA por hiperodoncias entre 2006 y 2010, 25 presentaron dientes supernumerarios y 3 presentaron odontomas.

De estos 25 pacientes, 8 eran niñas (32\%) y 17 eran niños (68\%).

Los 25 pacientes con dientes supernumerarios presentaban un total de 35 dientes supernumerarios.

\section{TABLA 1.- ENCUESTA TELEFÓNICA REALIZADA A LOS PACIENTES INTERVENIDOS POR SUPERNUMERARIOS EN EL HUCA ENTRE 2006 Y 2010}

1. ¿Tuvo algún problema de erupción de algún diente?
a. Previo a exodoncia
b. Postexodoncia

2. ¿'Tuvo que llevar ortodoncia?

3. De ser así, ćfue a causa de un problema generado por la presencia de supernumerarios?

4. ¿Se precisó tracción ortodóncica?

5. ¿Se resolvió el problema? 
De los 25 pacientes, 16 (64\%) presentaban un diente supernumerario único, 8 (32\%) tenían 2 supernumerarios y 1 paciente (4\%) tenía 3 (Figura 1 ).

Solo uno de los pacientes con supernumerarios presentaba hendidura labial bilateral, mientras el resto, no presentaban ninguna de las patologías que pueden estar relacionadas con la hiperodoncia mencionadas en la introducción.

De los 35 supernumerarios sólo uno de ellos era un diente primario o deciduo, 2,86\% del total de los supernumerarios.

Se presentaron con mayor frecuencia en la arcada superior, 32 de los supernumerarios $(91,43 \%)$, frente al $8,57 \%$ que se localizaban en la mandíbula.

La localización más frecuente fue la zona anterosuperior con 29 supernumerarios (82,86\% de los casos), de los cuales 12 eran mesiodens (34,29\%), 10 se encontraban en la zona incisivolateral $(28,57 \%)$ y 7 en la incisivocentral (20\%). Seguida de la zona premolar superior con 3 supernumerarios (8,57\%), de la zona premolar inferior con $2(5,71 \%)$ y por último, la región anteroinferior con 1 supernumerario $(2,86 \%)$.

La morfología solo era normal en 7 de los supernumerarios (20\%), el resto presentaban una morfología rudimentaria (80\%) (Figura 2).

De los 28 pacientes intervenidos en el HUCA se consiguió contactar telefónicamente con los 3 que presentaban odontomas y con 21 de los que presentaban supernumerarios.

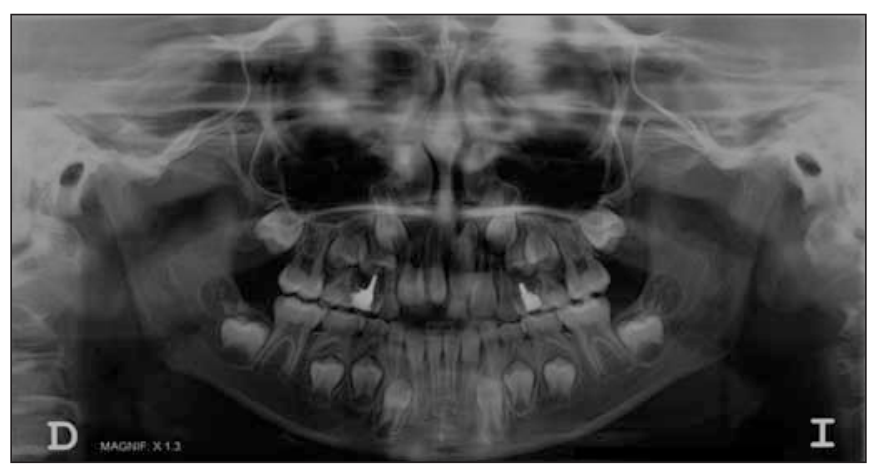

Fig. 1. Paciente con 2 dientes supernumerarios en frente anterosuperior.
De los 21 pacientes con dientes supernumerarios, $12(55,14 \%)$ respondieron que habían tenido problemas eruptivos previos a la exodoncia del/de los dientes supernumerarios y 9 de ellos mantenían esta situación tras la exodoncia.

Es decir, el $75 \%$ de los pacientes con problemas eruptivos previos presentan problemas eruptivos postexodoncia y representan un $42,86 \%$ del total de los pacientes.

Precisaron tratamiento ortodóncico a causa de hiperodoncia, 8 pacientes $(38,1 \%)$, de los cuales 3 $(37,5 \%)$ requirieron tracción y en otro de los pacientes se está valorando. Contestaron 2 pacientes, que están realizando revisiones periódicas a la espera de erupción espontánea, pero explicaron que muy probablemente comenzaran con tratamiento ortodóncico durante los próximos meses.

De estos 8 pacientes que requirieron tratamiento ortodóncico, uno de ellos presentaba 3 dientes supernumerarios, dos tenían 2 dientes supernumerarios y los cinco restantes 1 diente supernumerario. Todos ellos se localizaban en el frente anterosuperior (Tabla 2).

\section{DISCUSIÓN}

En este estudio la prevalencia de dientes supernumerarios es del $0,31 \%$ en la población infantil asturiana, menor si se compara tanto con los estudios sobre población caucásica $(10,17)$, como el realizado en población portuguesa, con una prevalencia

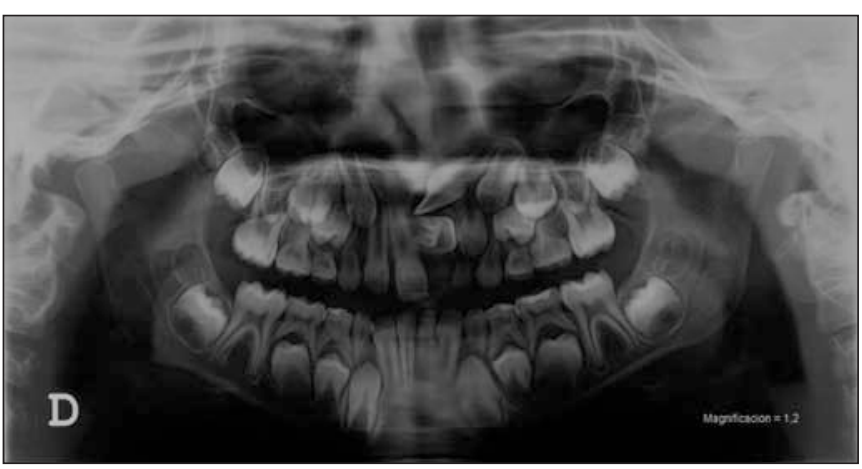

Fig. 2. Paciente con diente supernumerario en frente anterosuperior. 


\section{TABLA 2.- REPRESENTA EL NÚMERO DE SUPERNUMERARIOS Y SU LOCALIZACIÓN, ASÍ COMO LA NECESIDAD DE TRACCIÓN ORTODÓNCICA EN LOS OCHO PACIENTES QUE REQUIRIERON ORTODONCIA POR LA HIPERODONCIA}

\begin{tabular}{|c|c|c|c|}
\hline Paciente & $\begin{array}{c}\text { Número } \\
\text { de SN }\end{array}$ & Localización & $\begin{array}{c}\text { Tracción } \\
\text { ortodóncica }\end{array}$ \\
\hline 2 & 1 & 21 & No \\
\hline 8 & 1 & 22 & No \\
\hline 10 & 3 & $2(22)+$ mesiodens & $*$ \\
\hline 11 & 1 & Mesiodens & $\mathrm{Si}$ \\
\hline 13 & 2 & $21+$ mesiodens & $\mathrm{Si}$ \\
\hline 16 & 1 & Mesiodens & No \\
\hline 20 & 2 & 12,22 & $\mathrm{Si}$ \\
\hline 25 & 1 & Mesiodens & No \\
\hline * Se estaba valorando en el momento en que se realizó la encuesta. \\
\hline
\end{tabular}

del 0,76\% (13) o el realizado por Schmuckli en Suiza, con un $1,5 \%$ de prevalencia (18).

En línea coincidente con otros estudios, encontramos una mayor asociación al sexo masculino, en este caso con una ratio masculino/femenino de 2.125/1 $(18,19)$.

En estudios llevados a cabo por Esenlik (10) no se encontraron diferencias significativas en cuanto al sexo en un trabajo realizado en población turca; sin embargo, curiosamente, para esta misma población otros estudios hallaron un ratio masculino/femenino de $1,8 / 1$ (17).

La morfología de los supernumerarios fue fundamentalmente rudimentaria en el estudio que se realizó $(80 \%)$, ligeramente inferior a los resultados obtenidos por Patchett $(93,49 \%)$ (32).

El diente supernumerario único fue la forma más frecuente (64\%), seguido de 2 supernumerarios por paciente (32\%), similar a la del estudio de Ferrés-Padró con un $65,82 \%$ y $27,85 \%$, respectivamente (29).

Se coincide en este trabajo con estudios de otros autores en que la mayor parte de los supernumerarios se localizaron en la región anterosuperior $(82,6 \%)$ y la mayoría de ellos eran mesiodens $(34,29 \%)(10,14$, $15,18,29)$. En nuestro caso seguidos de los incisivos laterales superiores $(28,57 \%)$, de los incisivos centrales superiores (20\%), premolares superiores $(8,57 \%)$, premolares inferiores $(5,71 \%)$ y por último, de incisivos inferiores (2,86\%). Sin embargo, está distribución no fue igual entre los estudios revisados.

En este trabajo, el 55,14\% de los pacientes con dientes supernumerarios tuvieron problemas eruptivos previos a la exodoncia del/de los dientes supernumerarios y tres cuartas partes de ellos, el $42,86 \%$ del total de los pacientes, mantenían esta situación tras la exodoncia.

El 38,1\% de los pacientes de nuestro estudio precisaron tratamiento ortodóncico a causa del diente supernumerario, cifra muy similar a la obtenida por Patchetten los pacientes del Bristol Dental Hospital y a su vez inferior a la que obtuvieron estos mismos autores en el Westmead Dental Hospital con un $64,1 \%$ de pacientes que requirieron tratamiento ortodóncico, de estos pacientes con tratamiento ortodóncico, el 37,5\% requirieron de tracción mecánica.

Todos los pacientes de este trabajo que requirieron abordaje ortodóncico presentaban los dientes supernumerarios en la premaxila.

\section{CONCLUSIONES}

- La prevalencia de dientes supernumerarios en la población asturiana es baja y está más asociada al sexo masculino.

- Así mismo, son más frecuentes los dientes supernumerarios únicos y la morfología rudimentaria.

- Se localizan fundamentalmente en el frente anterosuperior.

- Con frecuencia, provocan retrasos eruptivos y retenciones dentarias que hacen imprescindible un diagnóstico precoz y que requiere muchas veces un tratamiento quirúrgico, asociado habitualmente, a un tratamiento ortodóncico que, en ocasiones, incluye la necesidad de tracción, especialmente cuando los dientes supernumerarios se sitúan en la premaxila. 


\section{BIBLIOGRAFÍA}

1. Tucker AS, Sharpe P. The cutting edge of mammalian development; how the embryo makes teeth. Nat Rev Genet 2004;5:499-508.

2. Fleming PS, Xavier GM, DiBiase AT, Cobourne MT. Revisiting the supernumerary the epidemiological and molecular basis of extra teeth. Br Dent J 2010; 208:25-30.

3. Canaval Torres C, Perea Paz M, Kanashiro Irakawa C, Mayo A. Dientes supernumerarios en odontopediatría. Rev Estomatol Heredian 2003;13(1-2):279.

4. Blanco G. Dientes multiples supernumerarios no relacinados a un síndrome: reporte de un caso. Revista Estomatología. 2005;13(1):13-9.

5. Kirmeier R, Truschnegg A, Payer M, Malyk J, Daghighi S, Jakse N. The supernumerary nasal tooth. Int J Oral Maxillofac Surg 2009;38(11): 1219-22.

6. Saleem T, Khalid U, Hameed A, Ghaffar S. Supernumerary, ectopic tooth in the maxillary antrum presenting with recurrent haemoptysis. Head Face Med 2010;6:26.

7. Sjöberg S, Lörinc P. Intracranial supernumerary tooth. Case report. Radiologe 1984;24(12):561-3.

8. Nagarajappa D, Manjunatha B. Tooth in oropharynx. J Oral MaxillofacPathol 2011;15(3): 346-7.

9. Grahnén H, Granath LE. Numerical variations in primary dentition and their correlation with the permanent dentition. Odont Rev 1961;12:348-57.

10. Esenlik E, Sayin MO, Atilla AO, Ozen C, Altun C, Başak F. Supernumerary teeth in a Turkish population. Am J Orthod Dentofacial Orthop 2009;136 (6): 848-52.

11. Rajab L, Hamdan M. Supernumerary teeth: Review of the literature and a survey of 152 cases. Int J Paediatr Dent 2002;12:244-54.
12. Ferriol-Fiol MN; Xavier S, Figueiredo R, Valmaseda-Castellón E, Gay-Escoda C. Hiperodoncia múltiple no asociada a síndromes. A propósito de dos casos. Med Oral Patol Oral Cir Bucal 2011; 16(Ed.esp.):89-93.

13. Costa-Pinho TM, Figueiredo-Pollmann MC. Study or the frecuency and the feature of supernumerary teeth found in one Portuguese population. Bull Group Int Rech Sci Stomatol Odontol 2004;46(23):52-62.

14. Fardi A, Kondylidou-Sidira A, Bachour Z, Parisis $N$, Tsirlis A. Incidence of impacted and supernumerary teeth- a radiographic study in a North Greek population. Med Oral Patol Oral Cir Bucal 2011;16(1):e56-61.

15. Küchler EC, Costa AG, Costa M de C, Vieira AR, Granjeiro JM. Supernumerary teeth vary depending on gender. Braz Oral Res 2011;25(1):76-9.

16. Salcido-García JF, Ledesma-Montes C, Hernández-Flores F, Pérez D, Garcés-Ortíz M. Frecuency of supernumerary teeth in Mexican population. Med Oral Patol Oral Cir Bucal 2004;9(5):407-9.

17. Mevlut M, Hasan K, Hüsametin O. Prevalence and characteristics of supernumerary teeth in a non-syndrome Turkish population: associated pathologies and proposed treatment. Med Oral Patol Oral Cir Bucal 2010;15(4):e575-8.

18. Schmuckli R, Lipowsky C, Peltomäki T. Prevalence and morphology of supernumerary teeth in the population of a Swiss community. Short communication. Schweiz Monatsschr Zahnmed 2010;120(11):987-93.

19. Leyland L, Batra P, Wong F, Llewelyn R. A retrospective evaluation of the eruption of impacted permanent incisors after extraction of supernumerary teeth. J Clin Pediatr Dent 2006; 30(3):225-31.

20. Wu TT, Chen PK, Lo LJ, Cheng MC, Ko EW. The characteristics and distribution of dental anomalies in patiens with cleft. Chang Gung Med J 2011;34 (3):306-14. 
21. Mehta DN, Vachhani RV, Patel MB. Cleidocranial dysplasia: A report of two cases. J Indian Soc Pedod Prev Dent 2011;29(3):251-4.

22. Acerbi AG, de Freitas C, Magalhães MH. Prevalence of numeric anomalies in the permanent dentition of patients with Down syndrome. Spec Care Dentist 2001;21(2):75-8.

23. Wolf J, Järvinen HJ, Hietanen J. Gardner's dento-maxillary stigmas in patients with familial adenomatosis coli. $\mathrm{Br} \mathrm{J}$ Oral Maxillofac Surg 1986;24(6):410-16.

24. Hibbert S.A previously unreported association between Nance-Horan syndrome and spontaneous dental abscesses.Oral Surg Oral Med Oral Pathol Oral RadiolEndod 2005;99(2):207-11.

25. Soneji B. Duplication of the mandibular primary dentition in orofacial-digital syndrome type IV. Cleft Palate Craniofac J 2010;47(3):259-63.

26. Kantaputra P, Miletich I, Lüdecke HJ, Suzuki EY, Praphanphoj V, Shivdasani R, et al. Trico- rhinophalangeal Syndrome with supernumerary teeth. J Dent Res 2008;87(11):1027-31.

27. Vadiati Saberi B, Shakoorpour A. Apert syndrome: report of a case with emphasis on oral manifestations. J Dent (Tehran) 2011; 8(2):90-5.

28. Khonsari RH, Corre P, Boykerma-Vernex Z, Schmidt J, Renaudin K, Frayssé C, et al. Extreme oral manifestations in a Marfan-type syndrome. Int J Oral Maxillofac Surg 2010;39(6):622-5.

29. Ferrés-Padró E, Prats-Armengol J, Ferrés-Amat E. A descriptive study of 113 unerupted supernumerary teeth in 79 pediatric patients in Barcelona. Med Oral Patol Oral Cir Bucal 2009;14(3): E146-52.

30. Rodríguez FJ, Cerviño S. Múltiples dientes supernumerarios distomolares. Av Odontoestomatol 2009;25(6):319-25.

31. Kalaskar RR, Kalaskar AR. Multidisciplinary management of impacted central incisors due to supernumerary teeth and associated dentigerous cyst. Contemp Clin Dent 2011;2(1):53-8.

32. Patchett CL, Crawford PJ, Cameron AC, Stephens $C D$. The management of supernumerary teeth in childhood - a retrospective study of practice in Bristol Dental Hospital, England and Westmead Dental Hospital, Sydney, Australia. Int J Paediatric Dent 2001;11:259-65.

\section{CORRESPONDENCIA}

Adolfo Pipa Vallejo

Escuela de Odontología.

Facultad de Medicina

Universidad de Oviedo

Correo electrónico: pipaadolfo@uniovi.es 